\title{
(Non-)convergence of solutions of the convective Allen-Cahn equation
}

\author{
Helmut Abels ${ }^{1}$ \\ Published online: 6 December 2021 \\ (C) The Author(s) 2021
}

\begin{abstract}
We consider the sharp interface limit of a convective Allen-Cahn equation, which can be part of a Navier-Stokes/Allen-Cahn system, for different scalings of the mobility $m_{\varepsilon}=m_{0} \varepsilon^{\theta}$ as $\varepsilon \rightarrow 0$. In the case $\theta>2$ we show a (non-)convergence result in the sense that the concentrations converge to the solution of a transport equation, but they do not behave like a rescaled optimal profile in normal direction to the interface as in the case $\theta=0$. Moreover, we show that an associated mean curvature functional does not converge to the corresponding functional for the sharp interface. Finally, we discuss the convergence in the case $\theta=0,1$ by the method of formally matched asymptotics.
\end{abstract}

Keywords Two-phase flow $\cdot$ Diffuse interface model $\cdot$ Allen-Cahn equation $\cdot$ Sharp interface limit

Mathematics Subject Classification 76T99 - 35Q30 - 35Q35 · 35R35 · 76D05 - 76D45

\section{Introduction}

In this contribution we consider the so-called sharp interface limit, i.e., the limit $\varepsilon \rightarrow 0$, of the convective Allen-Cahn equation

$$
\begin{aligned}
\partial_{t} c^{\varepsilon}+\mathbf{v} \cdot \nabla c^{\varepsilon} & =m_{\varepsilon}\left(\Delta c^{\varepsilon}-\varepsilon^{-2} f\left(c^{\varepsilon}\right)\right) \quad \text { in } \Omega \times(0, T), \\
\left.c^{\varepsilon}\right|_{\partial \Omega} & =-1 \quad \text { on } \partial \Omega \times(0, T), \\
\left.c^{\varepsilon}\right|_{t=0} & =c_{0}^{\varepsilon} \quad \text { in } \Omega .
\end{aligned}
$$

Here $\mathbf{v}: \Omega \times[0, T) \rightarrow \mathbb{R}^{d}$ is a given smooth divergence free velocity field with $\left.\mathbf{n} \cdot \mathbf{v}\right|_{\partial \Omega}=0$ and $c^{\varepsilon}: \Omega \times[0, T) \rightarrow \mathbb{R}$ is an order parameter, which will be close to the "pure states" \pm 1 for small $\varepsilon>0$. Here $f=F^{\prime}$, where $F: \mathbb{R} \rightarrow \mathbb{R}$ is a suitable double well potential with global minima \pm , e.g. $F(c)=\left(1-c^{2}\right)^{2} . c^{\varepsilon}$ can describe the concentration difference of

Dedicated to Prof. Hideo Kozono on the occasion of his 60th birthday

$凶$ Helmut Abels

helmut.abels@ur.de

1 Faculty of Mathematics, University of Regensburg, 93040 Regensburg, Germany 
two different phases in the case of phase transitions, where the total mass of each phase is not necessarily conserved. Moreover, $\Omega \subseteq \mathbb{R}^{d}$ is assumed to be a bounded domain with smooth boundary, $m_{\varepsilon}$ is a (constant) mobility coefficient and $\varepsilon>0$ is a parameter that is proportional to the "thickness" of the diffuse interface $\left\{x \in \Omega:\left|c^{\varepsilon}(x, t)\right|<1-\delta\right\}$ for $\delta \in(0,1)$.

The convective Allen-Cahn equation (1) is part of the following diffuse interface model for the two-phase flow of two incompressible Newtonian, partly miscible fluids with phase transition

$$
\begin{aligned}
\partial_{t} \mathbf{v}^{\varepsilon}+\mathbf{v}^{\varepsilon} \cdot \nabla \mathbf{v}^{\varepsilon}-\operatorname{div}\left(v\left(c^{\varepsilon}\right) D \mathbf{v}^{\varepsilon}\right)+\nabla p^{\varepsilon} & =-\varepsilon \operatorname{div}\left(\nabla c^{\varepsilon} \otimes \nabla c^{\varepsilon}\right), \\
\operatorname{div} \mathbf{v}^{\varepsilon} & =0, \\
\partial_{t} c^{\varepsilon}+\mathbf{v}^{\varepsilon} \cdot \nabla c^{\varepsilon} & =m_{\varepsilon}\left(\Delta c^{\varepsilon}-\varepsilon^{-2} f\left(c^{\varepsilon}\right)\right)
\end{aligned}
$$

in $\Omega \times(0, T)$, where $\mathbf{v}^{\varepsilon}: \Omega \times[0, T) \rightarrow \mathbb{R}^{d}$ is the velocity of the mixture, $D \mathbf{v}^{\varepsilon}=\frac{1}{2}\left(\nabla \mathbf{v}^{\varepsilon}+\right.$ $\left.\left(\nabla \mathbf{v}^{\varepsilon}\right)^{T}\right), p^{\varepsilon}: \Omega \times[0, T) \rightarrow \mathbb{R}$ is the pressure, and $v\left(c^{\varepsilon}\right)>0$ is the viscosity of the mixture. This model can be considered as a model for a two-phase flow with phase transition or an approximation of a classical sharp interface model for a two-phase flow of incompressible fluids with surface tension. Here the densities of the two separate fluids are assumed to be the same. A derivation of this model in a more general form with variable densities can be found in Jiang et al. [10]. We refer to Gal and Grasselli [6] for the existence of weak solutions and results on the longtime behavior of solutions for this model and to Giorgini et al. [7] for analytic results for a volume preserving variant with different densities. Mathematically, this system arises if one replaces the Cahn-Hilliard equation in the well-known "model H", cf. e.g. $[1,8]$, by an Allen-Cahn equation.

With the aid of formally matched asymptotic expansions one can formally show that solutions of this system converge to solutions of the following free boundary value problem

$$
\begin{aligned}
\partial_{t} \mathbf{v}+\mathbf{v} \cdot \nabla \mathbf{v}-\operatorname{div}\left(v^{ \pm} D \mathbf{v}\right)+\nabla p & =0 \quad \text { in } \Omega^{ \pm}(t), t \in(0, T), \\
\operatorname{div} \mathbf{v} & =0 \quad \text { in } \Omega^{ \pm}(t), t \in(0, T), \\
{[\mathbf{v}]_{\Gamma_{t}} } & =0 \quad \text { on } \Gamma_{t}, t \in(0, T), \\
-\left[\mathbf{n}_{\Gamma_{t}} \cdot\left(v^{ \pm} D \mathbf{v}-p \mathrm{Id}\right)\right]_{\Gamma_{t}} & =\sigma H_{\Gamma_{t}} \mathbf{n}_{\Gamma_{t}} \quad \text { on } \Gamma_{t}, t \in(0, T), \\
V_{\Gamma_{t}}-\mathbf{n}_{\Gamma_{t}} \cdot \mathbf{v} & =m_{0} H_{\Gamma_{t}} \quad \text { on } \Gamma_{t}, t \in(0, T),
\end{aligned}
$$

when $m_{\varepsilon}=m_{0}>0$ and

$$
\begin{aligned}
\partial_{t} \mathbf{v}+\mathbf{v} \cdot \nabla \mathbf{v}-\operatorname{div}\left(v^{ \pm} D \mathbf{v}\right)+\nabla p & =0 \quad \text { in } \Omega^{ \pm}(t), t \in(0, T), \\
\operatorname{div} \mathbf{v} & =0 \quad \text { in } \Omega^{ \pm}(t), t \in(0, T), \\
{[\mathbf{v}]_{\Gamma_{t}} } & =0 \quad \text { on } \Gamma_{t}, t \in(0, T), \\
-\left[\mathbf{n}_{\Gamma_{t}} \cdot\left(v^{ \pm} D \mathbf{v}-p \mathrm{Id}\right)\right]_{\Gamma_{t}} & =\sigma H_{\Gamma_{t}} \mathbf{n}_{\Gamma_{t}} \quad \text { on } \Gamma_{t}, t \in(0, T), \\
V_{\Gamma_{t}}-\mathbf{n}_{\Gamma_{t}} \cdot \mathbf{v} & =0 \quad \text { on } \Gamma_{t}, t \in(0, T),
\end{aligned}
$$

when $m_{\varepsilon}=m_{0} \varepsilon, m_{0}>0$. We will discuss this formal result in the appendix in more detail, cf. Remark 2 below. Here $v^{ \pm}>0$ are viscosity constants, $\Omega^{ \pm}(t) \subset \Omega$ are open and disjoint such that $\partial \Omega^{-}(t)=\Gamma_{t}=\partial \Omega^{+}(t) \cap \Omega, \mathbf{n}_{\Gamma_{t}}$ denotes the outer normal of $\partial \Omega^{-}(t)$ and the normal velocity and the mean curvature of $\Gamma_{t}$ are denoted by $V_{\Gamma_{t}}$ and $H_{\Gamma_{t}}$, respectively, taken with respect to $\mathbf{n}_{\Gamma_{t}}$. Furthermore, $[.]_{\Gamma_{t}}$ denotes the jump of a quantity across the interface in the direction of $\mathbf{n}_{\Gamma_{t}}$, i.e., $[f]_{\Gamma_{t}}(x)=\lim _{h \rightarrow 0}\left(f\left(x+h \mathbf{n}_{\Gamma_{t}}\right)-f\left(x-h \mathbf{n}_{\Gamma_{t}}\right)\right)$ for $x \in \Gamma_{t}$.

In the case $v^{+}=v^{-}$and that the Navier-Stokes equation is replaced by a (quasi-stationary) Stokes system Liu and the author proved rigorously in [4] that the convergence holds true in 
the first case $m_{\varepsilon}=m_{0}>0$ for sufficiently small times and for well-prepared initial data. More precisely, it was shown that in a neighborhood of $\Gamma_{t}$

$$
c_{\varepsilon}(x, t)=\theta_{0}\left(\frac{d_{\Gamma_{t}}(x)-\varepsilon h_{\varepsilon}(x, t)}{\varepsilon}\right)+\mathcal{O}(\varepsilon)
$$

(even with $\mathcal{O}\left(\varepsilon^{2}\right)$ ), where $d_{\Gamma_{t}}$ is the signed distance function to $\Gamma_{t}$ and $h_{\varepsilon}$ are correction terms, which are uniformly bounded in $\varepsilon \in(0,1)$, and $\theta_{0}: \mathbb{R} \rightarrow \mathbb{R}$ is the so-called optimal profile that is determined by

$$
-\theta_{0}^{\prime \prime}+f\left(\theta_{0}\right)=0 \text { in } \mathbb{R}, \quad \theta_{0}(0)=0, \quad \lim _{z \rightarrow \pm \infty} \theta_{0}(z)= \pm 1
$$

This form is important in order to obtain in the limit $\varepsilon \rightarrow 0$ the Young-Laplace law (15), cf. e.g. [2, Section 4].

It is the goal of the present contribution to show that in the case $m_{\varepsilon}=m_{0} \varepsilon^{\theta}$ with $\theta>2$ the solutions of the convective Allen-Cahn equation (1)-(2) do not have the form (17) in general. Moreover, we will show that the functional

$$
\left\langle H^{\varepsilon}, \varphi\right\rangle:=\varepsilon \int_{\Omega} \nabla c^{\varepsilon} \otimes \nabla c^{\varepsilon}: \nabla \varphi d x
$$

does not converge to the mean curvature functional

$$
\begin{aligned}
2 \sigma \int_{\Gamma_{t}} \mathbf{n}_{\Gamma_{t}} \otimes \mathbf{n}_{\Gamma_{t}}: \nabla \boldsymbol{\varphi} d \mathcal{H}^{d-1} & =-2 \sigma \int_{\Gamma_{t}}\left(\mathrm{Id}-\mathbf{n}_{\Gamma_{t}} \otimes \mathbf{n}_{\Gamma_{t}}\right): \nabla \boldsymbol{\varphi} d \mathcal{H}^{d-1} \\
& =-2 \sigma \int_{\Gamma_{t}} H_{\Gamma_{t}} \mathbf{n}_{\Gamma_{t}} \cdot \boldsymbol{\varphi} d \mathcal{H}^{d-1}
\end{aligned}
$$

for all $\varphi \in C_{0, \sigma}^{\infty}(\Omega)=\left\{f \in C_{0}^{\infty}(\Omega)^{d}: \operatorname{div} f=0\right\}$, where

$$
\sigma=\frac{1}{2} \int_{\mathbb{R}}\left(\theta_{0}^{\prime}(z)\right)^{2} d z
$$

We note that $H^{\varepsilon}$ is the weak formulation of the right-hand side of (4), which should converge to a weak formulation of the right-hand side of (15). Therefore there is no hope that solutions of the full system (4)-(6) converge to solutions of the corresponding limit system with (15) as $\varepsilon \rightarrow 0$ in the case that $m_{\varepsilon}=m_{0} \varepsilon^{\theta}, \theta>2$. We note that this effect was first observed for the corresponding Navier-Stokes/Cahn-Hilliard system by Schaubeck and the author in [5] in the case $\theta>3$. These results are also contained in the PhD-thesis of Schaubeck [11]. It is not difficult to show that $\left\langle H^{\varepsilon}, \varphi\right\rangle$ converges to (19) if (17) holds true in a sufficiently strong sense. Moreover, in the case $\theta>3$ non-convergence of the Navier-Stokes/CahnHilliard system in the case of radial symmetry and an inflow boundary condition was shown by Lengeler and the author in [3, Section 4]. We note that the latter counter example can be adapted to the present case of a Navier-Stokes/Allen-Cahn equation in the case $\theta>2$.

The structure of this contribution is as follows: in Sect. 2 we summarize some preliminaries and notation. Afterwards we prove the nonconvergence result in Sect. 3. Finally, in Sect. 4 we discuss briefly the sharp interface limit of the convective Allen-Cahn equation in the case $m_{\varepsilon}=m_{0} \varepsilon^{\theta}$ with $\theta=0,1$. 


\section{Preliminaries and notation}

We denote $a \otimes b=\left(a_{i} b_{j}\right)_{i, j=1}^{d}$ for $a, b \in \mathbb{R}^{d}$ and $A: B=\sum_{i, j=1}^{d} A_{i j} B_{i j}$ for $A, B \in \mathbb{R}^{d \times d}$. We assume that $\Omega \subset \mathbb{R}^{d}$ is a bounded domain with smooth boundary $\partial \Omega$. Furthermore, we define $\Omega_{T}=\Omega \times(0, T)$ and $\partial_{T} \Omega=\partial \Omega \times(0, T)$ for $T>0$. Moreover, $\mathbf{n}_{\partial \Omega}$ denotes the exterior unit normal on $\partial \Omega$. For a hypersurface $\Gamma_{t} \subset \Omega, t \in[0, T]$, without boundary such that $\Gamma_{t}=\partial \Omega^{-}(t)$ for a domain $\Omega^{-}(t) \subset \subset \Omega$, the interior domain is denoted by $\Omega^{-}(t)$ and the exterior domain by $\Omega^{+}(t):=\Omega \backslash\left(\Omega^{-}(t) \cup \Gamma_{t}\right)$, i.e., $\Gamma_{t}$ separates $\Omega$ into an interior and an exterior domain. $\mathbf{n}_{\Gamma_{t}}$ is the exterior unit normal on $\partial \Omega^{-}(t)=\Gamma_{t}$. The mean curvature of $\Gamma_{t}$ with respect to $\mathbf{n}_{\Gamma_{t}}$ is denoted by $H_{\Gamma_{t}}$. In the following $d_{\Gamma_{t}}$ is the signed distance function to $\Gamma_{t}$ chosen such that $d_{\Gamma_{t}}<0$ in $\Omega^{-}(t)$ and $d_{\Gamma_{t}}>0$ in $\Omega^{+}(t)$. By this convention we obtain $\nabla d_{\Gamma_{t}}=\mathbf{n}_{\Gamma_{t}}$ on $\Gamma_{t}$. Moreover, we define

$$
Q^{ \pm}:=\left\{(x, t) \in \Omega_{T}: d(x, t) \gtrless 0\right\} .
$$

The "double-well" potential $F: \mathbb{R} \rightarrow \mathbb{R}$ is a smooth function taking its global minimum 0 at \pm 1 . For its derivative $f(c)=F^{\prime}(c)$ we assume

$$
f( \pm 1)=0, \quad f^{\prime}( \pm 1)>0, \quad \int_{-1}^{u} f(s) d s=\int_{1}^{u} f(s) d s>0
$$

for all $u \in(-1,1)$. In Eq. (1) the given velocity field satisfies $\mathbf{v} \in C_{b}^{0}\left([0, T] ; C_{b}^{4}(\bar{\Omega})\right)^{d}$ with $\operatorname{div} \mathbf{v}=0$ and $\mathbf{v} \cdot \mathbf{n}_{\partial \Omega}=0$ on $\partial \Omega$ and the mobility constant $m_{\varepsilon}$ has the form $m_{\varepsilon}=m_{0} \varepsilon^{\theta}$ for some $\theta \geq 0$ and $m_{0}>0$. In Eq. (3) we choose the special initial value

$$
\left.c^{\varepsilon}\right|_{t=0}=\zeta\left(\frac{d_{\Gamma_{0}}}{\delta}\right) \theta_{0}\left(\frac{d_{\Gamma_{0}}}{\varepsilon}\right)+\left(1-\zeta\left(\frac{d_{\Gamma_{0}}}{\delta}\right)\right)\left(2 \chi_{\left\{d_{\Gamma_{0}} \geq 0\right\}}-1\right) \quad \text { in } \Omega,
$$

where we determine the constant $\delta>0$ later. Here $\zeta \in C_{0}^{\infty}(\mathbb{R})$ is a cut-off function such that

$$
\zeta(z)=1 \text { if }|z|<\frac{1}{2}, \quad \zeta(z)=0 \text { if }|z|>1, \quad z \zeta^{\prime}(z) \leq 0 \text { in } \mathbb{R},
$$

and $\theta_{0}$ is the unique solution to (18). This choice of the initial value is natural in view of (17).

\section{Nonconvergence result}

Our main result is:

Theorem 1 Let $\theta>2, \Omega \subset \mathbb{R}^{d}$ be a bounded domain with smooth boundary $\partial \Omega, \Gamma_{0} a$ smooth hypersurface such that $\Gamma_{0}=\partial \Omega_{0}^{-}$for a domain $\Omega_{0}^{-} \subset \subset \Omega$ and let $c^{\varepsilon}$ be the solution to the convective Allen-Cahn equation (1), (2) with initial condition (21). Then for every $T>0$ and for all $\varphi \in C^{\infty}\left([0, T] ; \mathcal{D}(\Omega)^{d}\right)$ with $\operatorname{div} \varphi=0$ we have

$$
\int_{0}^{T}\left\langle H^{\varepsilon}, \varphi\right\rangle d t \rightarrow_{\varepsilon \rightarrow 0} 2 \sigma \int_{0}^{T} \int_{\Gamma_{t}}\left|\nabla\left(d_{\Gamma_{0}}\left(X_{t}^{-1}\right)\right)\right| \mathbf{n}_{\Gamma_{t}} \otimes \mathbf{n}_{\Gamma_{t}}: \nabla \boldsymbol{\varphi} d \mathcal{H}^{d-1} d t .
$$

Here the evolving hypersurface $\Gamma_{t}, t \in[0, T]$, is the solution of the evolution equation

$$
V_{\Gamma_{t}}(x)=\mathbf{n}_{\Gamma_{t}}(x, t) \cdot \mathbf{v}(x, t) \text { for } x \in \Gamma_{t}, t \in(0, T], \quad \Gamma(0)=\Gamma_{0},
$$

where $V_{\Gamma_{t}}$ is the normal velocity of $\Gamma_{t}$, and $X_{t}: \Omega \rightarrow \Omega$ is defined by $X_{t}\left(y_{0}\right)=y\left(t ; y_{0}\right)$ for $y_{0} \in \Omega, t \in[0, T]$, where $y\left(\cdot ; y_{0}\right)$ is the solution of

$$
\frac{d}{d s} y\left(s ; y_{0}\right)=\mathbf{v}\left(y\left(s ; y_{0}\right), s\right), s \in[0, T], \quad y\left(0 ; y_{0}\right)=y_{0} .
$$


Moreover, it holds

$$
\left\|c^{\varepsilon}-\left(2 \chi_{Q^{+}}-1\right)\right\|_{L^{2}\left(\Omega_{T}\right)}^{2}=\mathcal{O}(\varepsilon) \text { as } \varepsilon \rightarrow 0 .
$$

Remark 1 In general $\left|\nabla\left(d_{\Gamma_{0}}\left(X_{t}^{-1}\right)\right)\right|=\left|D X_{t}^{-T} \nabla d_{\Gamma_{0}} \circ X_{t}^{-1}\right| \neq 1$, we refer to [5, Remark 1] for a proof. This shows that the weak formulation of $H^{\varepsilon}$ does not converge to the weak formulation of the right-hand side of the Young-Laplace law (15) in general.

To prove the theorem we follow the same strategy as in [5]: First we construct a family of approximate solutions $\left\{c_{A}^{\varepsilon}\right\}_{0<\varepsilon \leq 1}$. Afterwards we estimate the difference $\nabla\left(c^{\varepsilon}-c_{A}^{\varepsilon}\right)$, which will enable us to prove the assertion of the theorem. We start with the observation that $\Gamma_{t}:=X_{t}\left(\Gamma_{0}\right)$ is the solution to the evolution equation.

Lemma 1 Let $\Gamma_{0} \subset \Omega$ be a given smooth hypersurface such that $\Gamma_{0}=\partial \Omega_{0}^{-}$for a domain $\Omega_{0}^{-} \subset \subset \Omega$. Then the evolving hypersurface $\Gamma_{t}:=X_{t}\left(\Gamma_{0}\right) \subset \Omega, t \in[0, T]$, is the solution to the problem

$$
V_{\Gamma_{t}}=\mathbf{n}_{\Gamma_{t}} \cdot \mathbf{v} \text { on } \Gamma_{t}, t \in(0, T), \quad \Gamma(0)=\Gamma_{0} .
$$

We refer to [5, Lemma 3] for the proof.

For the following let $P_{\Gamma_{t}}(x)$ be the orthogonal projection of $x$ onto $\Gamma_{t}$. Then there exists a constant $\delta>0$ such that $\left.\Gamma_{t}(\delta):=\left\{x \in \Omega: \mid d_{\Gamma_{t}}(x)\right) \mid<\delta\right\} \subset \Omega$ and $\tau_{t}: \Gamma_{t}(\delta) \rightarrow$ $(-\delta, \delta) \times \Gamma_{t}$ defined by $\tau_{t}(x)=\left(d_{\Gamma_{t}}(x), P_{\Gamma_{t}}(x)\right)$ is a smooth diffeomorphism, cf. e.g. [9, Chapter 4.6].

We will need the following result:

Lemma 2 For $e: \bigcup_{t \in[0, T]} X_{t}\left(\Gamma_{0}(\delta)\right) \times\{t\} \rightarrow \mathbb{R}$ defined by $e(x, t):=d_{\Gamma_{0}}\left(X_{t}^{-1}(x)\right)$ the following properties hold:

1. $\frac{d}{d t} e(x, t)=-\mathbf{v}(x, t) \cdot \nabla e(x, t)$ for all $(x, t) \in \bigcup_{t \in[0, T]} X_{t}\left(\Gamma_{0}(\delta)\right) \times\{t\}$.

2. $e(x, t)$ is a level set function for $\Gamma_{t}$, i.e., $e(x, t)=0$ if and only if $x \in \Gamma_{t}$.

We refer to [5, Lemma 4] for the proof.

As mentioned in Sect. 2, let $\theta_{0}$ be the solution to (18) and let $\zeta$ be a cut-off function as in (22). Then we define

$$
c_{A}^{\varepsilon}(x, t):= \begin{cases} \pm 1 & \text { in } \overline{Q^{ \pm}} \cap \bigcup_{t \in[0, T]} \overline{X_{t}\left(\Omega \backslash \Gamma_{0}(\delta)\right)} \times\{t\}, \\ \zeta\left(\frac{e}{\delta}\right) \theta_{0}\left(\frac{e}{\varepsilon}\right) \pm\left(1-\zeta\left(\frac{e}{\delta}\right)\right) & \text { in } Q^{ \pm} \cap \bigcup_{t \in[0, T]} X_{t}\left(\Gamma_{0}(\delta) \backslash \Gamma_{0}\left(\frac{\delta}{2}\right)\right) \times\{t\}, \\ \theta_{0}\left(\frac{e}{\varepsilon}\right) & \text { in } \bigcup_{t \in[0, T]} X_{t}\left(\Gamma_{0}\left(\frac{\delta}{2}\right)\right) \times\{t\} .\end{cases}
$$

Then we have $c_{A}^{\varepsilon}(., 0)=c^{\varepsilon}(., 0)$ since $e(., 0)=d_{\Gamma_{0}}$ and

$$
\partial_{t} c_{A}^{\varepsilon}+\mathbf{v} \cdot \nabla c_{A}^{\varepsilon}=0 \text { in } \Omega_{T}
$$

since $\partial_{t} e+\mathbf{v} \cdot \nabla e=0$. Moreover, by the construction

$$
c_{A}^{\varepsilon}=0 \text { on } \partial \Omega \text {. }
$$

Furthermore, we define the approximate mean curvature functional by

$$
\left\langle H_{A}^{\varepsilon}, \boldsymbol{\varphi}\right\rangle=\varepsilon \int_{\Omega} \nabla c_{A}^{\varepsilon} \otimes \nabla c_{A}^{\varepsilon}: \nabla \varphi d x
$$

for all $\varphi \in \mathcal{D}(\Omega)^{d}$ with $\operatorname{div} \varphi=0$. Then we have: 
Lemma 3 Let $c_{A}^{\varepsilon}$ be defined as above. Then there exists some constant $C>0$ independent of $\varepsilon$ and $\varepsilon_{0} \in(0,1]$ such that the estimates

$$
\begin{aligned}
\left\|\Delta c_{A}^{\varepsilon}(., t)\right\|_{L^{2}(\Omega)} & \leq C \varepsilon^{-\frac{3}{2}}, \\
\left\|\nabla c_{A}^{\varepsilon}(., t)\right\|_{L^{2}(\Omega)} & \leq C \varepsilon^{-\frac{1}{2}}, \\
\left\|f\left(c_{A}^{\varepsilon}(., t)\right)\right\|_{L^{2}(\Omega)} & \leq C \varepsilon^{\frac{1}{2}} \\
\left\|c_{A}^{\varepsilon}(., t)-\left(2 \chi_{Q^{+}}(., t)-1\right)\right\|_{L^{2}(\Omega)} & \leq C \varepsilon^{\frac{1}{2}}
\end{aligned}
$$

hold for all $t \in[0, T]$ and $\varepsilon \in\left(0, \varepsilon_{0}\right)$.

We refer to [5, Lemma 5] for the proof.

Now we are able to prove the central lemma for the proof of Theorem 1.

Lemma 4 Let $c_{A}^{\varepsilon}$ be defined as above and let $c^{\varepsilon}$ be the unique solution to (1), (2) with initial condition (21). Then, for $\theta \geq 2$, there exists some constant $C>0$ independent of $\varepsilon$ and $\varepsilon_{0}>0$ such that

$$
\begin{aligned}
\varepsilon\left\|\nabla\left(c^{\varepsilon}-c_{A}^{\varepsilon}\right)\right\|_{L^{2}\left(\Omega_{T}\right)}^{2} & \leq C \varepsilon^{\theta-2} \text { and } \\
\left\|c^{\varepsilon}-c_{A}^{\varepsilon}\right\|_{L^{\infty}\left(0, T ; L^{2}(\Omega)\right)} & \leq C \varepsilon^{\theta-\frac{3}{2}}
\end{aligned}
$$

for all $\varepsilon \in\left(0, \varepsilon_{0}\right]$.

Proof First of all, we note that $c^{\varepsilon}(x, t), c_{A}^{\varepsilon}(x, t) \in[-1,1]$ for all $x \in \Omega, t \in(0, T)$. For $c_{A}^{\varepsilon}$ this follows from the construction and for $c^{\varepsilon}$ by the maximum principle.

We denote by $u=c^{\varepsilon}-c_{A}^{\varepsilon}$ the difference between exact and approximate solution, which solves

$$
\partial_{t} c_{A}^{\varepsilon}+\mathbf{v} \cdot \nabla c_{A}^{\varepsilon}=0 \text { in } \Omega_{T} .
$$

We multiply the difference of the differential equations for $c^{\varepsilon}$ and $c_{A}^{\varepsilon}$ by $u$ and integrate the resulting equation over $\Omega$. Then we get for all $t \in(0, T)$

$$
\begin{aligned}
0= & \int_{\Omega} u\left[\partial_{t} u+\mathbf{v} \cdot \nabla u-m_{0} \varepsilon^{\theta} \Delta u-m_{0} \varepsilon^{\theta} \Delta c_{A}^{\varepsilon}+m_{0} \varepsilon^{\theta-2} f\left(c^{\varepsilon}\right)\right] d x \\
= & \int_{\Omega}\left(\partial_{t} \frac{|u|^{2}}{2}-\mathbf{v} \cdot \nabla \frac{|u|^{2}}{2}+m_{0} \varepsilon^{\theta}|\nabla u|^{2}\right) d x \\
& +\int_{\Omega}\left(m_{0} \varepsilon^{\theta} \nabla u \cdot \nabla c_{A}^{\varepsilon}+m_{0} \varepsilon^{\theta-2} f\left(c^{\varepsilon}\right) u\right) d x \\
= & \frac{1}{2} \frac{d}{d t} \int_{\Omega}|u|^{2} d x+m_{0} \varepsilon^{\theta} \int_{\Omega}|\nabla u|^{2} d x-\int_{\Omega}\left(m_{0} \varepsilon^{\theta} u \Delta c_{A}^{\varepsilon}-m_{0} \varepsilon^{\theta-2} u f\left(c^{\varepsilon}\right)\right) d x,
\end{aligned}
$$

where we have used $u=0$ on $\partial \Omega$ as well as $\operatorname{div} \mathbf{v}=0$ in $\Omega$. By Hölder's and Young's inequalities we obtain

$$
\begin{aligned}
& \frac{1}{2} \frac{d}{d t} \int_{\Omega}|u|^{2} d x+\frac{m_{0}}{2} \varepsilon^{\theta} \int_{\Omega}|\nabla u|^{2} d x \\
& \quad \leq \frac{1}{2}\|u\|_{L^{2}(\Omega)}^{2}+\frac{m_{0}^{2} \varepsilon^{2 \theta}}{2}\left\|\Delta c_{A}^{\varepsilon}\right\|_{L^{2}(\Omega)}^{2}+m_{0} \varepsilon^{\theta-2}\left|\int_{\Omega} f\left(c^{\varepsilon}\right) u d x\right|
\end{aligned}
$$


for all $\varepsilon \in\left(0, \varepsilon_{0}\right)$, where

$$
\begin{aligned}
\left|\int_{\Omega} f\left(c^{\varepsilon}\right) u d x\right| & \leq\left|\int_{\Omega} f\left(c_{A}^{\varepsilon}\right) u d x\right|+C\|u\|_{L^{2}(\Omega)}^{2} \\
& \leq\left\|f\left(c_{A}^{\varepsilon}\right)\right\|_{L^{2}(\Omega)}\|u\|_{L^{2}(\Omega)}+C\|u\|_{L^{2}(\Omega)}^{2} \\
& \leq C \varepsilon^{\frac{1}{2}}\|u\|_{L^{2}(\Omega)}+C\|u\|_{L^{2}(\Omega)}^{2}
\end{aligned}
$$

since $f^{\prime}$ is Lipschitz continuous on $[-1,1]$. Hence (29) together with (30) and (23) yield

$$
\begin{aligned}
& \frac{1}{2} \frac{d}{d t} \int_{\Omega}|u|^{2} d x+m_{0} \varepsilon^{\theta} \int_{\Omega}|\nabla u|^{2} d x \\
& \quad \leq C\left(\|u\|_{L^{2}(\Omega)}^{2}+\varepsilon^{2 \theta-3}+\varepsilon^{\theta-2}\|u\|_{L^{2}(\Omega)}^{2}\right) \leq C_{1}\left(\|u\|_{L^{2}(\Omega)}^{2}+\varepsilon^{2 \theta-3}\right)
\end{aligned}
$$

since $\theta \geq 2$ for some $C_{1}>0$ independent of $\varepsilon$ and $t \in[0, T]$. Hence the Gronwall inequality implies

$$
\sup _{0 \leq t \leq T}\|u\|_{L^{2}(\Omega)}^{2}+\varepsilon^{\theta}\|\nabla u\|_{L^{2}((0, T) \times \Omega)}^{2} \leq C \varepsilon^{2 \theta-3}
$$

for some $C=C(T)>0$ independent of $\varepsilon$. Therefore the lemma is proved.

Now we can show that $H^{\varepsilon}-H_{A}^{\varepsilon}$ converges to 0 as $\varepsilon$ goes to zero.

Lemma 5 Let $H^{\varepsilon}$ and $H_{A}^{\varepsilon}$ be defined as above and let $\theta>2$. Then it holds

$$
\left|\int_{0}^{T}\left\langle H^{\varepsilon}-H_{A}^{\varepsilon}, \varphi\right\rangle d t\right| \rightarrow_{\varepsilon \rightarrow 0} 0,
$$

for all $\varphi \in C^{\infty}\left([0, T] ; \mathcal{D}(\Omega)^{d}\right)$.

Proof The proof is almost the same as in [5, Lemma 6]. But we include it for the convenience of the reader since the argument is central for our main result. Let $\varphi \in C^{\infty}\left([0, T] ; \mathcal{D}(\Omega)^{d}\right)$ and set $u=c^{\varepsilon}-c_{A}^{\varepsilon}$. Then

$$
\begin{aligned}
& \varepsilon\left|\int_{\Omega_{T}}\left(\nabla c^{\varepsilon} \otimes \nabla c^{\varepsilon}-\nabla c_{A}^{\varepsilon} \otimes \nabla c_{A}^{\varepsilon}\right): \nabla \varphi d x\right| \\
& \quad \leq \varepsilon\left|\int_{\Omega_{T}}\left(\nabla c^{\varepsilon} \otimes \nabla u\right): \nabla \varphi d x\right|+\varepsilon\left|\int_{\Omega_{T}}\left(\nabla u \otimes \nabla c_{A}^{\varepsilon}\right): \nabla \varphi d x\right| \\
& \quad \leq \varepsilon\|\nabla \varphi\|_{L^{\infty}\left(\Omega_{T}\right)}\|\nabla u\|_{L^{2}\left(\Omega_{T}\right)}\left(\left\|\nabla c^{\varepsilon}\right\|_{L^{2}\left(\Omega_{T}\right)}+\left\|\nabla c_{A}^{\varepsilon}\right\|_{L^{2}\left(\Omega_{T}\right)}\right) .
\end{aligned}
$$

Because of Lemmas 3 and 4, we have

$$
\left\|\nabla c^{\varepsilon}\right\|_{L^{2}\left(\Omega_{T}\right)} \leq\left\|\nabla c_{A}^{\varepsilon}\right\|_{L^{2}\left(\Omega_{T}\right)}+\|\nabla u\|_{L^{2}\left(\Omega_{T}\right)} \leq C\left(\varepsilon^{-\frac{1}{2}}+\varepsilon^{\frac{\theta-3}{2}}\right) .
$$

Using Lemma 4 we conclude

$$
\left|\int_{0}^{T}\left\langle H^{\varepsilon}-H_{A}^{\varepsilon}, \varphi\right\rangle d t\right| \leq C \varepsilon^{\frac{\theta-2}{2}}\left(1+\varepsilon^{\frac{\theta-2}{2}}\right)
$$

for some constant $C=C(\varphi)>0$ and for all $\varepsilon$ small enough. Since $\theta>2$, the assertion follows. 
Lemma 6 Let $H_{A}^{\varepsilon}$ and $c_{A}^{\varepsilon}$ be defined as above. Then it holds for all $\varphi \in \mathcal{D}(\Omega)^{d}$ and $t \in[0, T]$

$$
\left\langle H_{A}^{\varepsilon}, \varphi\right\rangle \rightarrow_{\varepsilon \rightarrow 0} 2 \sigma \int_{\Gamma_{t}}\left|\nabla\left(d_{\Gamma_{0}}\left(X_{t}^{-1}\right)\right)\right| \mathbf{n}_{\Gamma_{t}} \otimes \mathbf{n}_{\Gamma_{t}}: \nabla \boldsymbol{\varphi} d \mathcal{H}^{d-1}
$$

We refer to [5, Lemma 8] for the proof.

Proof of Theorem 1 The first assertion of the theorem immediately follows by Lemmas 5 and 6 . The second assertion is a consequence of Lemmas 3 and 4 since $\theta>2$.

\section{Formal asymptotics}

In this section we will use the method of formally matched asymptotic expansions to identify the sharp interface limit of the convective Allen-Cahn equation (1), (2) in the cases $m_{\varepsilon}=$ $m_{0} \varepsilon^{\theta}$ for $\theta=0,1$ and some $m_{0}>0$. We follow similar arguments as in [2, Section 4]. In particular we assume that there are smoothly evolving hypersurfaces $\Gamma_{t}, t \in(0, T)$, such that $\Gamma_{t}=\partial \Omega^{-}(t)$, and we have the following expansions: Outer expansion: "Away from $\Gamma_{t}$ " we assume that $c_{\varepsilon}$ has an expansion of the form:

$$
c_{\varepsilon}(x, t)=\sum_{k=0}^{\infty} \varepsilon^{k} c_{k}^{ \pm}(x, t) \text { for every } x \in \Omega^{ \pm}(t) .
$$

Inner expansion: In a neighborhood $\Gamma_{t}(\delta), \delta>0$, of $\Gamma_{t} c_{\varepsilon}$ has an expansion of the form:

$$
c_{\varepsilon}(x, t)=\sum_{k=0}^{\infty} \varepsilon^{k} c_{k}\left(\frac{d_{\Gamma_{t}}}{\varepsilon}, P_{\Gamma_{t}}(x), t\right) \text { for all } x \in \Gamma_{t}(\delta) .
$$

Matching condition:

$$
\begin{aligned}
\lim _{z \rightarrow \pm \infty} c_{k}(z, x, t) & =c_{k}^{ \pm}(x, t) \text { for all } x \in \Gamma_{t}, k=0,1, \\
\lim _{z \rightarrow \pm \infty} \partial_{z} c_{0}(z, x, t) & =0 \text { for all } x \in \Gamma_{t} .
\end{aligned}
$$

Moreover, all functions in the expansions above are assumed to be sufficiently smooth.

In the following we will use the expansions above and the matching conditions, insert them into the convective Allen-Cahn equation (1) and equate all terms of same order in order to determine the leading parts in the inner and outer expansions formally.

\subsection{Outer expansion}

First we use a power series expansion of $c_{\varepsilon}$ due to the outer expansion. Then

$$
f^{\prime}\left(c_{\varepsilon}(x, t)\right)=f^{\prime}\left(c_{0}^{ \pm}(x, t)\right) c_{1}^{ \pm}(x, t)+\varepsilon f^{\prime \prime}\left(c_{0}^{ \pm}(x, t)\right) c_{1}^{ \pm}(x, t)+\mathcal{O}\left(\varepsilon^{2}\right)
$$

and we obtain from (1)

$$
\frac{1}{\varepsilon^{2-k}} f^{\prime}\left(c_{0}^{ \pm}(x, t)\right)+\frac{1}{\varepsilon^{1-k}} f^{\prime \prime}\left(c_{0}^{ \pm}(x, t)\right) c_{1}^{ \pm}(x, t)+\mathcal{O}(1)=0
$$

for all $x \in \Omega^{ \pm}(t)$. This yields 
(i) At order $\frac{1}{\varepsilon^{2-k}}$ we obtain $f^{\prime}\left(c_{0}^{ \pm}(x, t)\right)=0$. Thus $c_{0}^{ \pm}(x, t) \in\{ \pm 1,0\}$. Here we exclude the case $c_{0}^{ \pm}(x, t)=0$ since 0 is unstable and define $\Omega^{ \pm}(t)$ such that

$$
c_{0}^{ \pm}(x, t)= \pm 1 \text { for all } x \in \Omega^{ \pm}(t) .
$$

(ii) If $k=0$, we obtain at order $\frac{1}{\varepsilon}$ that $f^{\prime \prime}\left(c_{0}(x, t)\right) c_{1}^{ \pm}(x, t)=0$. Since $f^{\prime \prime}( \pm 1)>0$, we conclude

$$
c_{1}^{ \pm}(x, t)=0 \text { for all } x \in \Omega^{ \pm}(t) .
$$

If $k=1$, the corresponding term is of order $\mathcal{O}(1)$ and we do not use this information. Moreover, we will not determine $c_{1}^{ \pm}$and $c_{1}$ in this case.

\subsection{Inner expansion}

In $\Gamma_{t}(\delta)$ we use the inner expansion in (1) in order to determine the leading coefficients $c_{0}(\rho, s, t)$ and, in the case $k=0, c_{1}(\rho, s, t)$, where $s:=s(x):=P_{\Gamma_{t}}(x)$. To this end we use

$$
\begin{aligned}
\mathbf{v} \cdot \nabla c_{j}(\rho, s, t) & =\frac{1}{\varepsilon} \mathbf{v} \cdot \nabla d_{\Gamma_{t}}(\rho, s, t)+\mathcal{O}(1), \\
\Delta c_{j}(\rho, s, t) & =\frac{1}{\varepsilon^{2}}\left(\partial_{\rho}^{2} c_{j}\right)(\rho, s, t)+\frac{1}{\varepsilon}\left(\partial_{\rho} c_{j}\right)(\rho, s, t) \Delta d_{\Gamma_{t}}(x)+\mathcal{O}(1), \\
\partial_{t} c_{j}(\rho, s, t) & =\frac{1}{\varepsilon}\left(\partial_{\rho} c_{j}\right)(\rho, s, t) \partial_{t} d_{\Gamma_{t}}(x)+\mathcal{O}(1)
\end{aligned}
$$

on $\Gamma_{t}$, where $\rho=\frac{d_{\Gamma_{t}}(x, t)}{\varepsilon}$ and

$$
\nabla d_{\Gamma_{t}}=\mathbf{n}_{\Gamma_{t}}, \quad \Delta d_{\Gamma_{t}}=-H_{\Gamma_{t}}, \quad \partial_{t} d_{\Gamma_{t}}=-V_{\Gamma_{t}} \text { on } \Gamma_{t} .
$$

Hence inserting the inner expansion in (1) and equating terms of the same order yields for all $x \in \Gamma_{t}$ :

$$
\begin{aligned}
& m_{0}\left[-\partial_{\rho}^{2} c_{0}(\rho, s, t)+f^{\prime}\left(c_{0}(\rho, s, t)\right)\right] \cdot \frac{1}{\varepsilon^{2}} \\
& \quad+m_{0}\left[-\partial_{\rho}^{2} c_{1}(\rho, s, t)+f^{\prime \prime}\left(c_{0}(\rho, s, t)\right) c_{1}(\rho, s, t)\right] \cdot \frac{1}{\varepsilon} \\
& \quad+\left[-\partial_{\rho} c_{0}(\rho, s, t)\left(V_{\Gamma_{t}}-\mathbf{n}_{\Gamma_{t}} \cdot \mathbf{v}-m_{0} H_{\Gamma_{t}}\right)\right] \cdot \frac{1}{\varepsilon}=O(1)
\end{aligned}
$$

in the case $k=0$ and

$$
\left[m_{0}\left(-\partial_{\rho}^{2} c_{0}(\rho, s, t)+f^{\prime}\left(c_{0}(\rho, s, t)\right)\right)-\left(\partial_{\rho} c_{0}\right)(\rho, s, t)\left(V_{\Gamma_{t}}-\mathbf{n}_{\Gamma_{t}} \cdot \mathbf{v}\right)\right] \cdot \frac{1}{\varepsilon}=O(1)
$$

in the case $k=1$. For the following we distinguish the cases $k=0,1$.

Case $k=0:$ The $\mathcal{O}\left(\frac{1}{\varepsilon^{2}}\right)$-terms yield

$$
-\partial_{\rho}^{2} c_{0}(\rho, s, t)+f^{\prime}\left(c_{0}(\rho, s, t)\right)=0 \text { for all } \rho \in \mathbb{R}, s \in \Gamma_{t}, t \in[0, T] .
$$

Because of the matching condition, we obtain

$$
\lim _{\rho \rightarrow \pm \infty} c_{0}(\rho, s, t)=c_{0}^{ \pm}(s, t)= \pm 1 \quad \text { for all } s \in \Gamma_{t}, t \in[0, T] .
$$


In order to obtain that $\Gamma_{t}$ approximates the zero-level set of $c_{\varepsilon}(x, t)=c_{0}\left(\frac{d_{\Gamma_{t}}}{\varepsilon}, s(x), t\right)+\mathcal{O}(\varepsilon)$ sufficiently well, we obtain $c_{0}(0, s, t)=0$. Hence

$$
c_{0}(\rho, x, t)=\theta_{0}(\rho) \text { for all } x \in \Gamma_{t}, \rho \in \mathbb{R} .
$$

Furthermore, the $\mathcal{O}\left(\frac{1}{\varepsilon}\right)$-terms yield

$$
m_{0}\left(-\partial_{\rho}^{2} c_{1}(\rho, x, t)+f^{\prime \prime}\left(\theta_{0}(\rho)\right) c_{1}(\rho, x, t)\right)=\theta_{0}^{\prime}(\rho)\left(V_{\Gamma_{t}}-\mathbf{n}_{\Gamma_{t}} \cdot \mathbf{v}-m_{0} H_{\Gamma_{t}}\right)=: g(\rho)
$$

Since $\theta_{0}^{\prime}$ is in the kernel of the differential operator $-\partial_{\rho}^{2}+f^{\prime \prime}\left(\theta_{0}\right)$, this ODE has a bounded solution if and only if

$$
\int_{\mathbb{R}} g(\rho) \theta_{0}^{\prime}(\rho) d \rho=0
$$

which is equivalent to

$$
V_{\Gamma_{t}}-\mathbf{n}_{\Gamma_{t}} \cdot \mathbf{v}=H_{\Gamma_{t}} \quad \text { on } \Gamma_{t} .
$$

Now the matching condition yields $c_{1}(\rho, x, t) \rightarrow_{\rho \rightarrow \pm \infty} c_{1}^{ \pm} \equiv 0$. Hence $c_{1} \equiv 0$ since the solution is unique. Altogether we obtain for the inner expansion

$$
c_{\varepsilon}(x, t)=\theta_{0}\left(\frac{d_{\Gamma_{t}}(x)}{\varepsilon}\right)+O\left(\varepsilon^{2}\right)
$$

close to $\Gamma_{t}$.

Case $k=1$ : The $\mathcal{O}\left(\frac{1}{\varepsilon}\right)$-terms yield

$$
\begin{aligned}
& m_{0}\left(-\partial_{\rho}^{2} c_{0}(\rho, s, t)+f^{\prime}\left(c_{0}(\rho, s, t)\right)\right) \\
& \quad-\partial_{\rho} c_{0}(\rho, s, t)\left(V_{\Gamma_{t}}(s)-\mathbf{n}_{\Gamma_{t}}(s) \cdot \mathbf{v}(s, t)\right)=0
\end{aligned}
$$

for all $s \in \Gamma_{t}$. Testing with $\partial_{\rho} c_{0}(\rho, x, t)$ yields

$$
0=\int_{\mathbb{R}}\left|\partial_{\rho} c_{0}(\rho, s, t)\right|^{2} d \rho\left(V_{\Gamma_{t}}(s)-\mathbf{n}_{\Gamma_{t}} \cdot \mathbf{v}(s, t)\right)
$$

since

$$
\int_{\mathbb{R}} \partial_{\rho}\left(\frac{\left|\partial_{\rho} c_{0}(\rho, s, t)\right|^{2}}{2}+f\left(c_{0}(\rho, s, t)\right)\right) d \rho=0
$$

because of the matching condition for $\partial_{\rho} c_{0}$. Because of $c_{0}(\rho, s, t) \rightarrow_{\rho \rightarrow \pm \infty} \pm 1, \partial_{\rho} c_{0}$ does not vanish and we obtain

$$
V_{\Gamma_{t}}=\mathbf{n}_{\Gamma_{t}} \cdot \mathbf{v} \quad \text { on } \Gamma_{t} .
$$

Moreover, we obtain from (32)

$$
-\partial_{\rho}^{2} c_{0}(\rho, s, t)+f^{\prime}\left(c_{0}(\rho, s, t)\right)=0 \text { for all } s \in \Gamma_{t}, \rho \in \mathbb{R} .
$$

Hence we can conclude as in the case $k=0$ that $c_{0}(\rho, s, t)=\theta_{0}(\rho)$ for all $\rho \in \mathbb{R}$ and $s \in \Gamma_{t}$, $t \in[0, T]$.

Remark 2 The formal calculations show that $c_{\varepsilon}$ should have an expansion of the form (17) in the case $\theta=0,1$. This is important to obtain (15) in the limit. Actually, using $c_{0}(\rho, s, t)=$ $\theta_{0}(\rho)$ one can easily modify the results in [2, Section 4] to show formally convergence of the Navier-Stokes/Allen-Cahn system (4)-(6) to (7)-(11) in the case $\theta=0$ and (12)-(16) in the case $\theta=1$. A rigorous justification of this convergence under suitable assumptions remains open. 
Acknowledgements The author is grateful to the anonymous referees for their careful reading and comments to improve the manuscript.

Funding Open Access funding enabled and organized by Projekt DEAL.

\section{Declarations}

Conflict of interest The author declares that he has no conflict of interest.

Open Access This article is licensed under a Creative Commons Attribution 4.0 International License, which permits use, sharing, adaptation, distribution and reproduction in any medium or format, as long as you give appropriate credit to the original author(s) and the source, provide a link to the Creative Commons licence, and indicate if changes were made. The images or other third party material in this article are included in the article's Creative Commons licence, unless indicated otherwise in a credit line to the material. If material is not included in the article's Creative Commons licence and your intended use is not permitted by statutory regulation or exceeds the permitted use, you will need to obtain permission directly from the copyright holder. To view a copy of this licence, visit http://creativecommons.org/licenses/by/4.0/.

\section{References}

1. Abels, H.: On a diffuse interface model for two-phase flows of viscous, incompressible fluids with matched densities. Arch. Rat. Mech. Anal. 194(2), 463-506 (2009)

2. Abels, H., Garcke, H., Grün, G.: Thermodynamically consistent, frame indifferent diffuse interface models for incompressible two-phase flows with different densities. Math. Models Methods Appl. Sci. 22(3), 1150013 (2012)

3. Abels, H., Lengeler, D.: On sharp interface limits for diffuse interface models for two-phase flows. Interfaces Free Bound. 16(3), 395-418 (2014). https://doi.org/10.4171/IFB/324

4. Abels, H., Liu, Y.: Sharp interface limit for a Stokes/Allen-Cahn system. Arch. Ration. Mech. Anal. 229(1), 417-502 (2018). https://doi.org/10.1007/s00205-018-1220-x

5. Abels, H., Schaubeck, S.: Nonconvergence of the capillary stress functional for solutions of the convective Cahn-Hilliard equation. In: Mathematical fluid dynamics, present and future. In: Springer Proceedings of Mathematical Statistics, vol. 183, pp. 3-23. Springer, Tokyo (2016)

6. Gal, C.G., Grasselli, M.: Longtime behavior for a model of homogeneous incompressible two-phase flows. Discrete Contin. Dyn. Syst. 28(1), 1-39 (2010). https://doi.org/10.3934/dcds.2010.28.1

7. Giorgini, A., Grasselli, M., Wu, H.: Diffuse interface models for incompressible binary fluids and the mass-conserving Allen-Cahn approximation. Preprint. arXiv:2005.07236 (2020)

8. Gurtin, M.E., Polignone, D., Viñals, J.: Two-phase binary fluids and immiscible fluids described by an order parameter. Math. Models Methods Appl. Sci. 6(6), 815-831 (1996)

9. Hildebrandt, S.: Analysis 2. Springer-Lehrbuch. [Springer Textbook]. Springer, Berlin (2003)

10. Jiang, J., Li, Y., Liu, C.: Two-phase incompressible flows with variable density: an energetic variational approach. Discrete Contin. Dyn. Syst. 37(6), 3243-3284 (2017). https://doi.org/10.3934/dcds.2017138

11. Schaubeck, S.: Sharp interface limits for diffuse interface models. Ph.D. thesis, University Regensburg, urn:nbn:de:bvb:355-epub-294622 (2014) 